\title{
Development and Application of Heat-Conductor Technique for Single Crystal Components of Superalloys
}

\author{
Dexin Ma ${ }^{1, \mathrm{a}}$, Bin Zhou ${ }^{2, \mathrm{~b}}$, Andreas Bührig-Polaczek ${ }^{1, \mathrm{c}}$ \\ ${ }^{1}$ Foundry Institute, RWTH University Aachen, Germany \\ ${ }^{2}$ Departmemt MTM, Katholieke Universiteit Leuven, Belgium
}

ad.ma@gi.rwth-aachen.de, bbin.zhou@mtm.kuleuven.be, 'sekretariat@gi.rwth-aachen.de

Key words: Superalloy, directional solidification, single crystal, heat-conductor

\begin{abstract}
During single crystal (SC) solidification in turbine blades of superalloys, grain defects often form in the platform region with abrupt variation in cross-section. In order to reduce grain defects induced by component geometries, a Heat Conductor (HC) technique was developed and applied to the production of SC turbine blades. The corresponding ceramic shell moulds were produced by a modified investment casting procedure. The heat conductors with excellent heat conductivity were inserted close to the inner corners of the platform, to effectively extract the local heat during directional solidification. Both computer simulation and temperature measurements have shown that this technique is able to produce a clear improvement of the thermal condition in the critical region of the components. The SC growth in the blade body can spread into the platform more quickly before the melt at the extremity becomes deeply undercooled. Microstructure investigations reveal a remarkable reduction in grain defect formation, providing confirmation of the effectiveness of the $\mathrm{HC}$ technique in improving the casting quality of SC components.
\end{abstract}

\section{Introduction}

Single crystal solidification of turbine blades for advanced gas turbines is a key technology for the production of reliable and high efficiency gas turbines. During the past years the established technology for aero-engines has been transferred to stationary gas turbines. With increasing component size the casting defects increases more than proportionally, the result is very low yield $[1,2]$. This is mainly due to the ineffective heat extraction caused by the thick shell mould with very low thermal conductivity. Especially at cross sectional transitions from blade to platform, the heat extraction becomes extremely inhomogeneous due to the ceramic accumulations during shell mould manufacturing. In this case, it is difficult to maintain a favourable temperature gradient at the liquid-solid interface for single crystal growth. It is known that stray grains in SC parts are related to curvature of the liquidus isotherm while it is passing through enlargements in the cross-section of the component (e.g. the platform of a turbine blade) [3,4]. As the solidification front approaches the platform, a significant degree of undercooling develops in the liquid at the extremity. As the front passes the mould corner, lateral growth of secondaries from the original primary dendrite into the platform is very fast due to the high undercooling. This can generate a complex branching pattern of dendrite structure. The high amount of latent heat released during this rapid growing phase can possibly result in partial remelting of small dendrite arms, leading to the formation of interdendritic equiaxed grains. When there is sufficient undercooling exceeding the undercoolability of the used alloy, new grains may nucleate to generate macroscopic stray grain defects [3-6]. This problem cannot be effectively overcome by conventional process modification such as optimization of the withdrawal rate. The LMC (liquid metal cooling) [2, 7, 8] and GCC (gas cooling casting) processes $[9,10]$ have been developed to increase the cooling rate in castings. The mould surface is no longer cooled by heat radiation, but more effectively by metal melt or inert gas flow. However, the key problem of inhomogeneous heat extraction from geometrical features still remains unsolved, or will probably become a more important issue. The heat resistance of the shell mould, which depends directly on mould thickness, becomes the limiting factor for heat transfer. In this case, the extremities of the platforms cool even more quickly than the inner corners due to the great difference in local mould thickness. This means that the liquidus isotherm becomes more concave and the stray grains form more easily at the platform extremities. In this paper a heat-conductor (HC) technique will be introduced, in order to effectively improve the thermal condition at the cross transition and hence to suppress the stray grain formation at extremities of the platform. 


\section{The Heat-Conductor (HC) technique}

The stray grain is formed preferentially in the abrupt cross-section transition of a component. This is attributed to the local thermal condition during directional solidification process. As shown in Figure 1a, the inner corner A of the platform cools much more slowly than at extremity B. This "hot spot" at position A slows down the advance of solidification front from the blade body into the platform. As a result the extremity B becomes an isolated undercooled region. While the local undercooling continuously increases, the undesired nucleation of a new grain may occur.

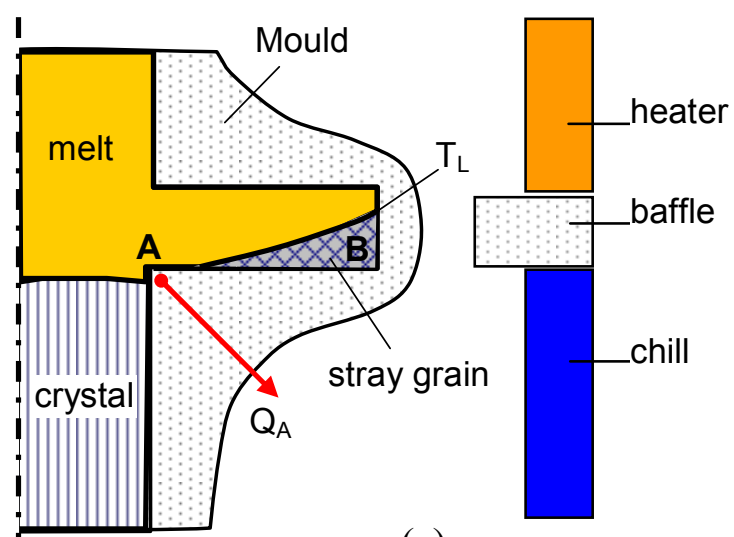

(a)

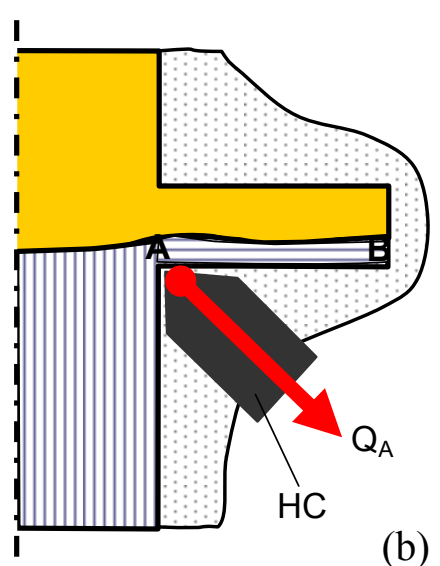

(b)

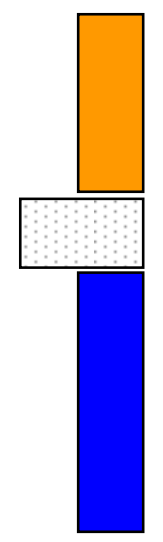

Figure 1: Schematic showing the formation of the undercooled zone and stray grain at the platform extremity B (a) and Principle of heat-conductor technique (b).

To overcome this problem, Heat-Conductor (HC) technique has been developed [11-13]. As illustrated in Figure 1b, the heat conductor can be inserted to the inner corner of the platform. Due to the significant improvement of the heat extraction $\mathrm{Q}_{\mathrm{A}}$, the hot spot at position $\mathrm{A}$ should be minimized. This favours the successful transition of single crystal growth from blade body into the extremity B before the local undercooling exceeds the critical value for equiaxed grain nucleation.

The $\mathrm{HC}$ technique is easy to apply. The technique necessitates no changes to the existing Bridgman set-up, but only a modification to shell mould manufacture, to insert the HC into the shell mould. Because of its refractory properties in a vacuum and its excellent thermophysical properties, graphite is the best material for the HC. The graphite has a minor disadvantage. During firing the shell mould cluster at high temperature, the inserted graphite block tends to react with the atmosphere, so some necessary protection measure has to be taken. Another well known heat conductor, $\mathrm{SiC}$, was also investigated in this research. The biggest advantage of $\mathrm{SiC}$ is its stability at high temperature without reaction with the atmosphere, so that the shell moulds with HCs can be simply fired without any protective measures. The conductivity of $\mathrm{SiC}$ is not as high as graphite, but still significantly better than that of the ceramic shell mould. Another disadvantage of $\mathrm{SiC}$ is that it is a hard material and relatively difficult to tailor to the required shape.

\section{Manufacture of ceramic shell moulds}

The most important procedure in this technique is the insertion of the $\mathrm{HC}$ into the ceramic shell moulds. For this purpose some methods have been developed in our research works:

\section{Method 1: bonding HC-block to the first layer of the shell mould}

This method, as principally shown in Fig. 2, was first used in our early research. After the assembly of the wax cluster, in which the blades were arranged in a circle around a central rod, the shell mould was made by dipping in ceramic slurries and sanding with fused alumina, as the standard casting. After the manufacture of the first and second layer the HCs were bonded at the locations selected using ceramic binder. The existing ceramic layer of approx. half a millimeter prevents contact and thus any reaction between the melt and the HCs. By repeating the procedure of dipping 
and sanding, the shell moulds were produced to the final thickness of approx. $8 \mathrm{~mm}$. Parallelly, the exterior of the $\mathrm{HC}$ was cleaned with a toothbrush to keep it free of ceramic accumulation. After drying the shell mould is dewaxed in a steam autoclave. The firing process of the shell moulds at high temperature should be carried out in a protective atmosphere to protect the HCs against oxidation. In our experience, however, the shell moulds can be fired in a closed furnace with some pieces of waste graphite. This protective measure has been proven to ensure that no vacuum and inert gas atmosphere is necessary. If $\mathrm{SiC}$ is used as $\mathrm{HC}$ material, the shell moulds can be fired simply in the air without any protective measure since $\mathrm{SiC}$ is stable at high temperature.

Method 1: Attaching $\mathrm{HC}$ after finishing the first ceramic layer
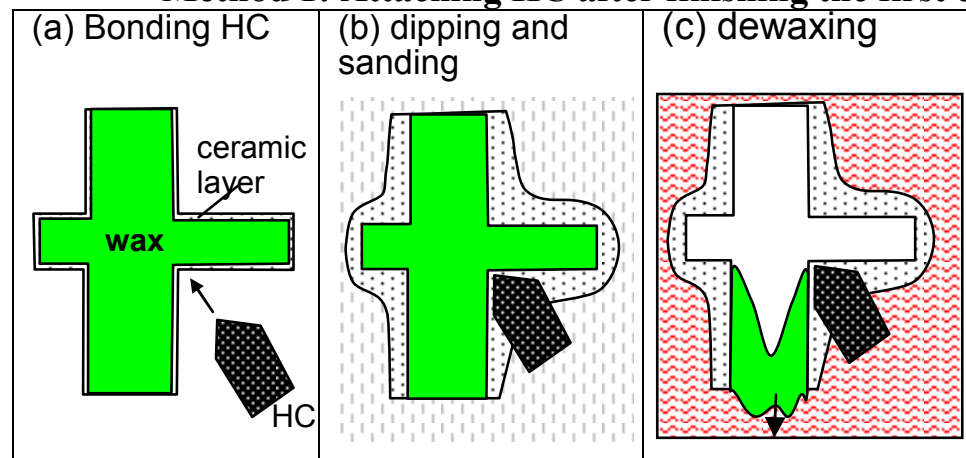

(d) Firing with protective measure

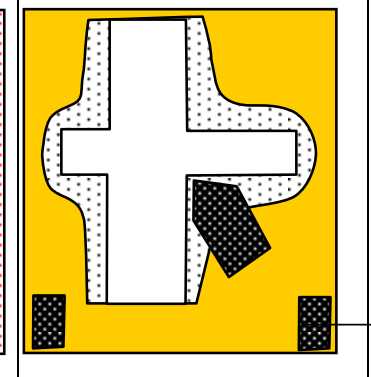

waste graphite

Method 2: Insertion of HC block in the preserved holes after firing

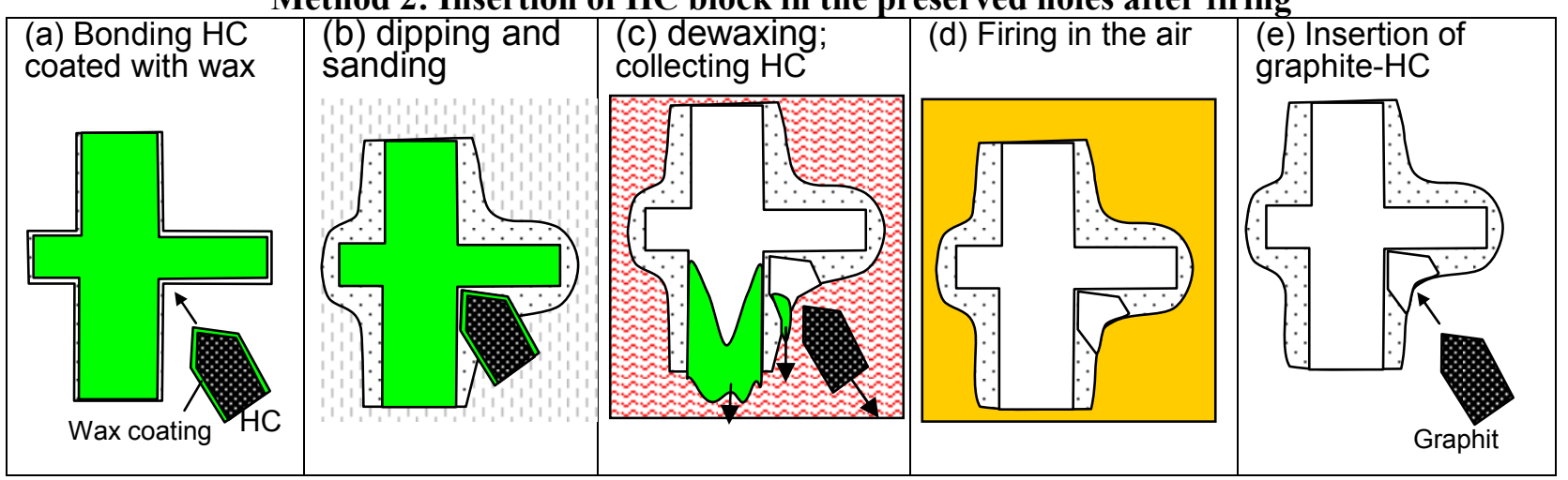

Method 3: Filling HC-Paster in the preserved holes after firing

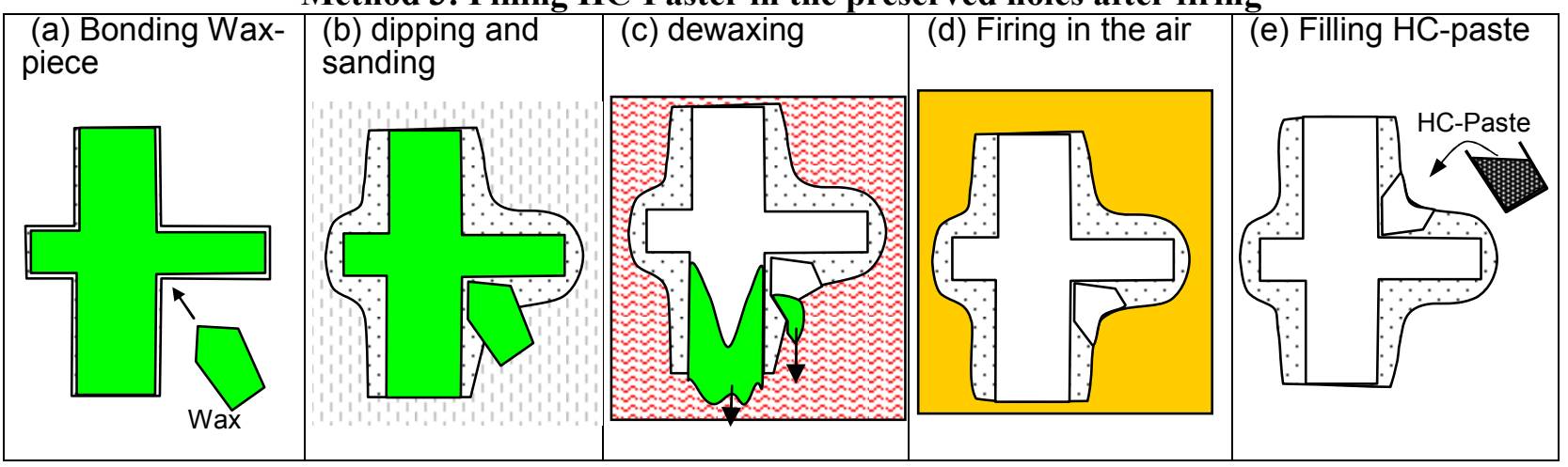

Fig. 2: Schematic illustration of procedures of shell mould manufacture for inserting HC.

\section{Method 2: Insertion of HC-block in the preserved holes after firing}

Prior to bonding HCs to the mould, the surface of the shaped graphite-HC should be coated with a thin wax film by dipping it into hot wax. The manufacture of the shell mould by dipping and sanding is the same as described in method 1. After dewaxing the HCs can be taken out because the wax film is melted away. Due to the absence of the graphite-HCs, the shell moulds can be fired as conventionally done without any protective measure. After firing process the graphite-HCs can be inserted into the preserved holes of the shell moulds (Fig.2, method 2). 


\section{Method 3: Filling HC-Paste in the preserved holes after firing}

The wax blocks in the shape of the $\mathrm{HC}$ can be attached after the manufacture of the first or second layer of the shell mould. During dewaxing of the produced shell mould in a steam autoclave the wax blocks were melted away and only holes of the shape of the HC remained. After firing of the mould the paste of graphite was inserted into the holes designed in shell moulds so as to obtain the HCs at the defined positions. Alternatively, the preserved holes can also be inserted with the HCblocks. However, the HC-blocks can only be inserted into the simple holes whose interior is not thicker than the exterior. In contrast, HC-paste can be used to form HC of any shape needed for optimal cooling of the critical location.

\section{Examination of $\mathrm{HC}$ technique}

In order to investigate the effect of the $\mathrm{HC}$, a dummy blade geometry featuring a shroud-like crosssection was designed (Fig. 3a). The projection of the platform is about $20 \mathrm{~mm}$. In half of the components, $\mathrm{HC}$ was applied so as to investigate its effectiveness. The casting experiments were performed following the standard Bridgman process. The alloy used in this experiment is a Nibased SC superalloy CMSX-6 (Cr 9.8, Co 5.0, Mo 3.0, Al 4.8 Ti 4.7 and Ta 2.0 in wt.\%). A heater temperature of $1500{ }^{\circ} \mathrm{C}$ and a withdrawal velocity of $3 \mathrm{~mm} / \mathrm{min}$ are standard process parameters of the Bridgman process. The measured cooling curves of the extremities are characterized by an undercooling $\Delta \mathrm{T}$ in the melt prior to the start of solidification (Fig. 3a).

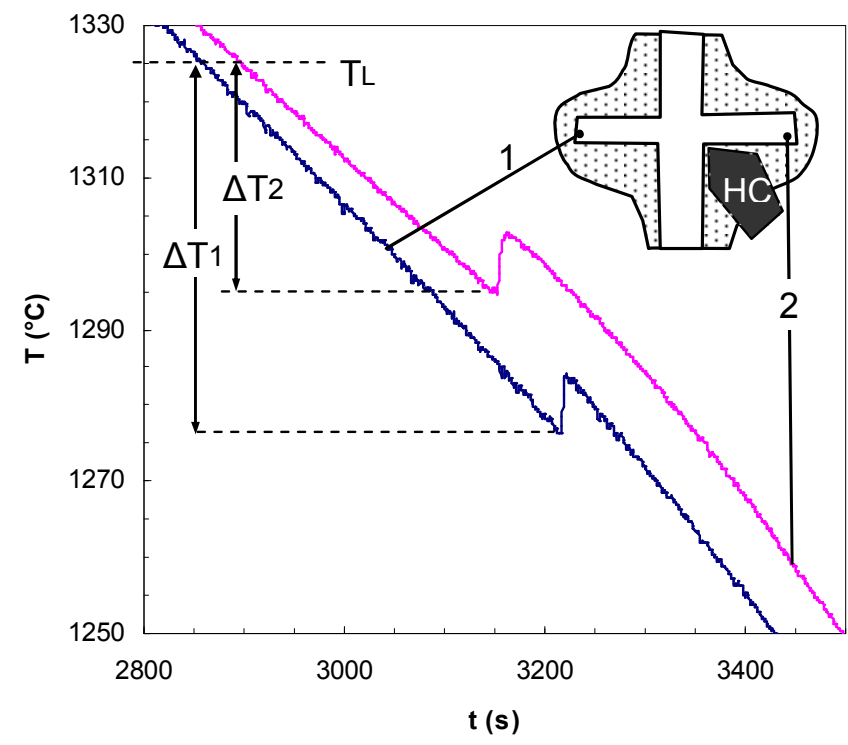

(a)

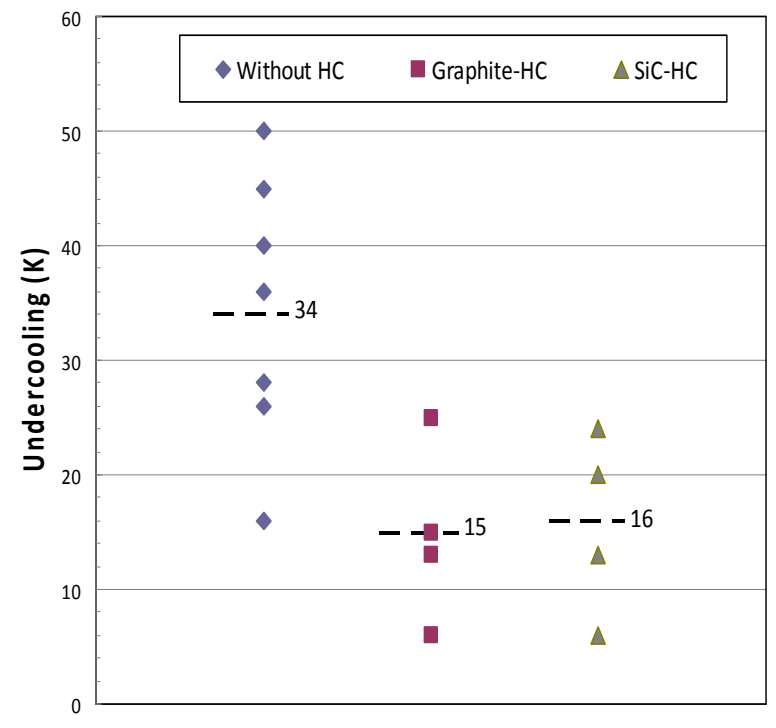

(b)

Fig. 3: (a) typical cooling curves measured at the platform extremities, without and with HC application;

(b) Overview of measured undercooling values at the platform extremities

(Broken line gives the average undercooling value).

Fig. 3a shows the typical cooling curves measured at the extremities of the platforms. Without HC application, an undercooling $\Delta \mathrm{T}_{1}$ of approx. $45 \mathrm{~K}$ below the liquidus temperature $\mathrm{T}_{\mathrm{L}}$ was measured before the local melt began to solidify. Applying HC significantly improves the local cooling condition. Thus, the undercooled zone at the extremity is no longer so isolated from the solidification front in the blade body. The undercooling $\Delta \mathrm{T}_{2}$ is significantly smaller.

Fig. 3b summarises the measured undercoolings at the extremities of the platforms with and without HCs. The results show a dramatic reduction of undercooling through application of either graphite or SiC HCs. Thus, both graphite and SiC HCS can be applied in the production of SC turbine blades. Since the probability of grain nucleation in an alloy melt is strongly dependent on the value of $\Delta \mathrm{T}$, the notable reduction achieved by applying $\mathrm{HC}$ will result in a significant reduction in the formation of stray grains. 
A further investigation was carried out for an actual blade geometry whose platform has both abrupt cross section enlargement from the aerofoil and an overhanging outer corner (Fig. 4). This design was selected to provide a typical geometrical feature which is the most prone to thermal undercooling and hence to subsequent grain defects. In this case, the application of the HC technique is especially desirable.

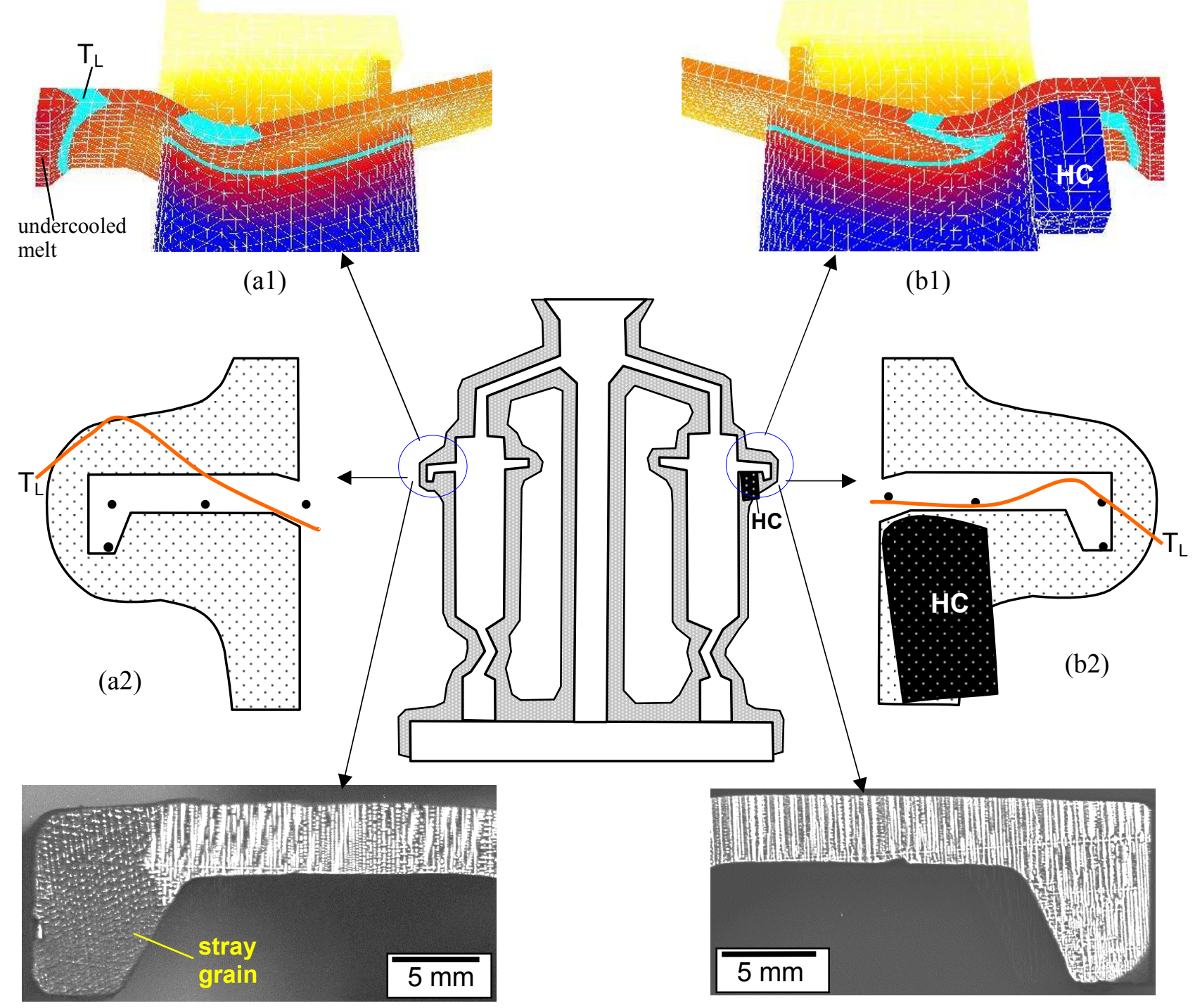

(a3)

(b3)

Fig. 4: Investigation with actual blade geometry. (a1, b1): Simulated temperature development in the blade platform; (a2, b2): Reconstructed liquidus-isotherm $\left(\mathrm{T}_{\mathrm{L}}\right)$ when passing through the platform; (a3, b3): microstructure of the platform.

Fig. 4-a1 and 4-b1 shows the simulated temperature development in two platforms, without (left) and with (right) application of $\mathrm{HC}$ respectively. The simulation result clearly shows the unavoidability of the thermal undercooling at the extremities and, at the same time, the effectiveness of the $\mathrm{HC}$ technique. Heat extraction from the cross-sectional transition is significantly improved. The solidification front in the blade body can move into the shroud more quickly, before the melt in the outer corner becomes deeply undercooled. From this prediction it can be assumed that the risk of heterogeneous nucleation of stray grain is dramatically reduced, although it remains impossible to eliminate undercooling entirely. The attached $\mathrm{HC}$ acts as a heat sink and effectively removes the hot barrier between the blade body and the extremity. The solidification front can then move into the shroud promptly, before the melt at the extremity becomes deeply undercooled. 
From the temperature measurement it is possible to reconstruct the liquidus isotherm passing through the platform (Fig. 4-a2 and 4-b2). With no HC, a significantly concave-shaped isotherm is obtained (except on the outer side where the isotherm becomes convex, because it is in a favourable position to radiation by the heaters). Through the application of $\mathrm{HC}$, the $\mathrm{T}_{\mathrm{L}}$-isotherm in the platform becomes significantly smoothed. Thus, the application of $\mathrm{HC}$ can significantly reduce the duration and the extent of the isolated undercooling, and consequently the possibility of grain defects.

The examination of as-cast structure shows that the platform extremities are very prone to grain defect formation due to the geometrical discontinuity and the subsequent isolated undercooling. Without using HC the majority of blades cast in this study are rejects due to the equiaxed grain structure (Fig. 4-a3). In contrast, nearly all blades with applying HC are acceptable because no high angle boundaries are observed (Fig. 4-b3). This confirms evidently the effectiveness of HC technique to reduce the grain defects caused by the geometric feature.

\section{Summary}

The heat conductor (HC) technique was developed and applied to produce SC components of superalloys. Some methods to insert the HC into ceramic shell moulds were introduced. In addition to graphite, $\mathrm{SiC}$ can be also selected as $\mathrm{HC}$ material since the shell moulds can be fired simply without any need to protect the inserted HC from reactions with the atmosphere. The graphite HCs can be inserted into the prefabricated holes in shell moulds after firing.

The effectiveness of the $\mathrm{HC}$ technique was investigated with the help of computer simulation, thermal measurements and metallographic examinations. The result shows that both graphite and $\mathrm{SiC} \mathrm{HC}$ are able to significantly reduce the undercooling at the isolated undercooled extremities Thus, the probability of stray grain nucleation at the extremities of the platforms is greatly reduced. As confirmed by metallographic investigations, the formation of stray at the platform extremities was effectively suppressed by application of the $\mathrm{HC}$ technique. This provides clear evidence of the effectiveness of the $\mathrm{HC}$ technique in improving the quality of SC components.

\section{References}

[1] B.B. Seth: Superalloys 2000, T.M. Pollock et al. (Eds.), TMS (2000) 3

[2] A.J. Elliott, S. Tin, W.T. King, S.C. Huang, M.F.X. Gigliotti, T.M. Pollock: Met. Mat. Trans. A, 35A (2004) 3221

[3] U. Paul, P.R. Sahm, D. Goldschmidt: Mat. Sci. Eng. A, 173 (1993) 49

[4] M. Meyer ter Vehn, D. Dedecke, U. Paul, P.R. Sahm: Superalloys 1996, R.D.Kissinger et al. (Eds.), TMS (1996) 471

[5] R.E. Napolitano, R.J. Schaefer: J .Mat. Sci. 35 (2000) 1641

[6] W. Wang, A. Kermanpur, P.D. Lee, M. Meclean: J. Mat. Sci. 38 (2003) 4385

[7] R.F. Singer: 3rd Symposium on Advanced Technologies and Processes for Metals and Alloys, 1995, Frankfurt

[8] F. Hugo: Patent DE 19730637 A1, 1997

[9] E.L. Kats, M. Konter, J. Rösler, V.P. Lubenets: Patent DE 19539770 A1 and US 5.921.310

[10] M. Konter, E. Kats, N. Hofmann: Superalloys 2000, T.M. Pollock et al. (Eds.) TMS (2000) 189

[11] D. Ma, A. Bührig-Polaczek: Patent DE 102007014744, 2007

[12] D. Ma, A. Bührig-Polaczek: Int. J. Cast Metals Res., 22 (2009) 422

[13] D. Ma, A. Bührig-Polaczek: Mel. Mat. Trans. B 40 (2009) 738 